\title{
CONVEX BODIES ASSOCIATED WITH A CONVEX BODY
}

\author{
PRESTON C. HAMMER
}

1. Introduction. In considering some hypotheses concerning the centroid of a convex body we were led to an interesting class of convex bodies associated with a given body. While a subclass is mentioned by B. H. Neumann, ${ }^{1}$ so far as we know the complete class has not heretofore been defined and its properties discussed. In making the proofs of our theorems we conceived the notion of a family of essential diameters of a convex body which has some interest apart from the use we have made of this family.

2. Notation. A theorem of B. H. Neumann. We shall confine our theorems to closed convex bodies in the plane. Generalization will be discussed later. The convex bodies will be denoted by capitals $A, B$, or $C$. The corresponding boundaries will be denoted by $\alpha, \beta$, or $\gamma$ respectively. Points in the plane will be designated by lower case letters $a, b, c, x, y$, or $z$. Real numbers occur as such in connection with ratios only and these will be designated by $r$. Straight lines are denoted by $m$ or $n$ and a line segment (directed when necessary) by its pair of end points. A line which contains at least one boundary point of a convex body $C$ and no interior points of the body we call a contact line. Such a line either meets $C$ in one boundary point or it contains a line segment in the boundary of $C$ and no other points of $C$. A diameter of $C$ is any chord the end points of which lie in a pair of parallel contact lines of $C$. Through an interior point $x$ of a convex body $C$ let a chord of $C$ be passed. $x$ divides the chord in some ratio $r$. For our purposes we take the ratio $r$ to be the ratio of the larger (or equal) segment to the whole chord. Then for a given $x$ the ratio has a maximum value for all chords. This maximum ratio we call $r(x)$. Extending the function $r(x)$ to the boundary $\gamma$ of $C$, we let $r(x)=1$ for all points $x$ on the boundary. Then $1 / 2 \leqq r(x) \leqq 1$ and $r(x)$ is a continuous function of the points $x$ over the closed set $C$. These maximum ratios then have a minimum value which we call the critical ratio of $C$ and designate by $r^{*}$. Concerning this ratio, B. H. Neumann proved the following theorem.

THEOREM. The value $r^{*}$ is assumed for a closed planar convex body

Presented to the International Congress of Mathematicians, August 31, 1950; received by the editors September 28, 1950.

${ }^{1}$ On some affine invariants of closed convex regions, J. London Math. Soc. vol. 14, p. 262. 
$C$ at a unique critical point $x^{*}$ interior to $C$. There are at least three chords of $C$ which $x^{*}$ divides in the ratio $r^{*}$. The range of $r^{*}$ for all convex planar bodies is $1 / 2 \leqq r^{*} \leqq 2 / 3$. The convex bodies for which $r^{*}=1 / 2$ have central convex curves for boundaries and those for which $r^{*}=2 / 3$ are triangles in which the centroid is $x^{*}$.

It is clear that the critical ratio and critical point are affine invariants of the convex body. We give elsewhere ${ }^{2}$ a generalizable proof of the inequality of Neumann's theorem.

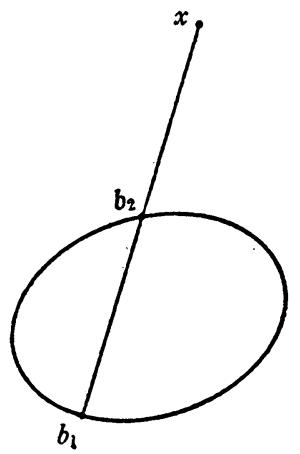

Fig. 1

3. Definitions of associated sets. Let $b$ be any boundary point of a convex body $C$ and let $r$ be any positive real number. The convex set obtained from $C$ by a similitude transformation with a ratio $r$ about center $b$ we designate by $C_{b}(r)$. Observe that if $r>1, C_{b}(r) \supset C$; if $r=1, C_{b}(r)=C$; and if $r<1, C_{b}(r) \subset C$. The associated sets $C(r)$ we define as follows:

1. If $r<1, C(r)=\prod_{b \in r} C_{b}(r)$.

2. If $r>1, C(r)=\sum_{b \in \gamma} C_{b}(r)$.

3. If $r=1, C=C(1)=\prod C_{b}(r)=\sum C_{b}(r)$.

Hence for every ratio $r>0$ we have a set $C(r)$ associated with $C$.

4. Essential diameters. Before stating and proving theorems concerning the associated sets defined in $\$ 3$ we consider the diameters of a planar convex body. If a straight line contains at least two distinct points of $C$, the chord of $C$ along that line is the intersection of

2 P. C. Hammer, The centroid of a convex body, Proceedings of the American Mathematical Society vol. 2 (1951) pp. 522-525.

${ }^{3} \mathrm{~W}$. Süss first gave a generalization of the inequality of Neumann's theorem in a paper entitled Ueber eine Affininvariante von Eibereichen, Archive der Mathematik vol. 1 (1948) pp. 127-128. A minor correction to Süss' statement of results can be found in the reference of footnote 2 . 
the line with $C$. If two parallel lines $m_{1}$ and $m_{2}$ each contain at least one boundary point of the body $C$ but no interior points of $C$, then all chords of $C$ joining boundary points of $C$ respectively on $m_{1}$ and $m_{2}$ are called diameters of $C$. Concerning these diameters we now state a few theorems.

THEOREM 1. Every point $x$ in the plane exterior to $C$ lies on at least one extended diameter of $C$.

Proof. Let $x$ be a point exterior to $C$ and consider a ray through $x$

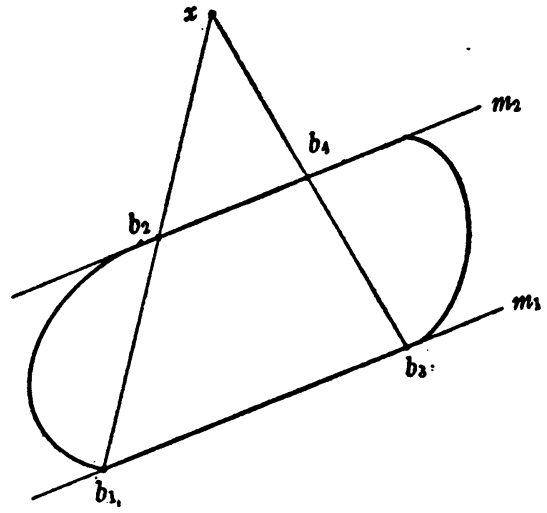

FIG. 2

intersecting $C$ in boundary points $b_{2}$ and $b_{1}$ so that $b_{1} x$ contains $b_{2}$ (see Fig. 1). In particular, $b_{2}$ may coincide with $b_{1}$ on a contact ray or $b_{1} b_{2}$ may be a chord of $C$ on its boundary. The ratio $b_{1} b_{2} / b_{1} x$ varies continuously as a function of the angle the segment $b_{1} x$ makes with a fixed direction between the "contact" directions. Hence the ratio must assume a maximum value. It can then be readily shown that the ray for which the ratio is a maximum is an extended diameter of $C^{4}$

Theorem 2. (1) If a point $x$ exterior to $C$ lies on two distinct extended diameters of $C$, then there is a pair of parallel line segments in the boundary of $C$ which contain respective end points of the diameters extending through $x$.

(2) If two diameters of $C$ are parallel, there exists a pair of parallel line segments in the boundary of $C$ containing the respective end points of each of the parallel diameters.

Proof. To prove part (1), let any two diameters of $C$ through $x$ be given (Fig. 2). Let $b_{1} b_{2}$ be one diameter and let $m_{1}$ and $m_{2}$ be parallel

'See Appendix A for a proof suggested by the referee. 
contact lines of $C$ passing through $b_{1}$ and $b_{2}$ respectively. Then $C$ lies in the closed strip $m_{1} m_{2}$. Hence, the end points $b_{3}$ and $b_{4}$ of the second diameter of $C$ which extends through $x$ must be in the strip. Now, as the line segment $b_{1} b_{2}$ lies in $C$, the only possible location of $b_{3}$ and $b_{4}$ is on the lines $m_{1}$ and $m_{2}$, for otherwise it would be impossible to have two parallel contact lines of $C$ through $b_{3}$ and $b_{4}$. Then $b_{2} b_{4}$ and $b_{1} b_{3}$ are parallel line segments in the boundary of $C$. It follows that any other diameter extending through $x$ has its end points on $m_{1}$ and $m_{2}$. Obviously there are then infinitely many diameters of $C$ through $x$. The proof of part (2) is quite analogous to that of part (1).

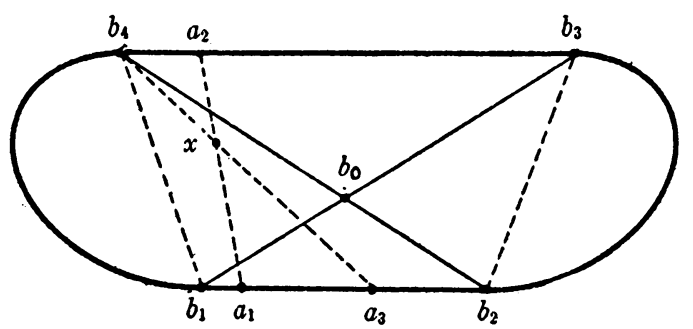

FIG. 3

THEOREM 3. There exists a unique family $F$ of diameters of $C$ with the following properties:

(1) Every point $x$ exterior to $C$ lies on exactly one diameter of $F$ extended.

(2) To every direction in the plane there corresponds exactly one diameter in $F$ with that direction.

(3) Each diameter in $F$ intersects every other diameter in $F$ in $C$.

(4) Every point in $C$ is on at least one diameter of $F$.

(5) The cumulated positive area swept out in $C$ by diameters of $F$ as a function of a direction angle is a minimum among all families satisfying properties (1)-(4).

Proof of Theorem 3. From Theorems 1 and 2 it follows that if an exterior point $x$ lies on a unique extended diameter of $C$, then every point exterior to $C$ on that infinite extended diameter lies only on that extended diameter. Now let $\beta$ be a circle with $C$ in its interior. If to any point $x$ on $\beta$ there is a unique diameter of $C$ which extends through it, we assign that diameter to $F$. If for any point $x$ on $\beta$ there are two diameters of $C$ which extend through it, by Theorem 2 there is a maximal pair of parallel chords $b_{1} b_{2}$ and $b_{3} b_{4}$ in the boundary of $C$ such that respective end points of all diameters extending through $x$ are on $b_{1} b_{2}$ and $b_{3} b_{4}$. Here the points $b_{1} b_{2} b_{3} b_{4}$ (see Fig. 3 ) are taken in 
a counterclockwise sense on the boundary of $C$. The region outside $C$ covered by extending diameters with respective end points on $b_{1} b_{2}$ and $b_{3} b_{4}$ is determined by these chords and the extended diameters $b_{1} b_{3}$ and $b_{2} b_{4}$. Furthermore, no point in this region lies on any other extended diameter of $C$ from the proof of Theorem 2. The diameters $b_{1} b_{3}$ and $b_{2} b_{4}$ intersect in a point interior to $C$. We now assign diameters to $F$ if their end points respectively lie on $b_{1} b_{2}$ and $b_{3} b_{4}$ and if they pass through the intersection point of $b_{1} b_{3}$ and $b_{2} b_{4}$.

This assignment of diameters gives a simple coverage, by extensions, of the region exterior to $C$ which was multiply covered by extensions of all diameters. Our method of assigning diameters to $F$ is completely described. Every point exterior to $C$ is simply covered by diameters in $F$. Consider the transformation from $x$ to $y$ where $x$ and $y$ are on the same extended diameter of $C$ and both are on the circle $\beta$. If any other diameter in $F$ extends to intersect $\beta$ in points $a$ and $b$, then $a$ and $b$ separate $x$ and $y$ on $\beta$; for every two diameters in $F$ intersect in $C$ since they are not parallel and cannot intersect by extension exterior to $C$. Hence it follows that $y$ is a continuous image of $x$ and as $x$ proceeds in a counterclockwise sense on $\beta$, so does $y{ }^{5}$ This implies that every direction in the plane is assumed once by diameters in $F$ and as the extended diameters $x, y$ cover the circular disk, every point in $C$ is on at least one. This concludes the proof of properties 1, 2, 3, and 4 .

Property 5 is demonstrated if we show that our assignment of diameters for parallel chords in the boundary leads to a minimal area among all families of diameters satisfying properties (1)-(4). Referring to Fig. 3, we see that the area swept out in $C$ by diameters in $F$ with respective end points in $b_{1} b_{2}$ and $b_{3} b_{4}$ is the area in the two triangles $b_{0} b_{1} b_{2}$ and $b_{0} b_{3} b_{4}$ where $b_{0}$ is the intersection point of $b_{1} b_{3}$ and $b_{2} b_{4}$. It can be shown that for any other family of diameters satisfying properties (1)-(4) the assigned diameters with respective end points in $b_{1} b_{2}$ and $b_{3} b_{4}$ must cover the two triangles and that only the choice we made leads to a minimum area. Hence the family $F$ of diameters is uniquely determined by the conditions of Theorem 3 .

Definition. Every diameter of $C$ belonging to $F$ as defined above is called an essential diameter of $C$. All other diameters we call extraneous. The term essential will be more completely justified in later theorems.

Corollary to Theorems 2 and 3. If a convex body has no pair of

\footnotetext{
5 The relationship between $x$ and $y$ should be more properly described as an involutory transformation of the circle onto itself without fixed points.
} 
parallel line segments in its boundary, the family of essential diameters coincides with the family of all diameters.

Theorem 4. A point $x$ interior to $C$ divides at least one essential diameter in the ratio $r=r(x)$.

Proof. First of all, if $x$ divides a chord of $C$ in its maximum ratio, then that chord is a diameter of $C$; for otherwise "nearby" chords of $C$ through $x$ would be divided by $x$ in a ratio greater than $r .{ }^{6}$ Let $a_{1} a_{2}$ be a diameter of $C$ which $x$ divides in a ratio $r$, specifically $a_{1} x / a_{1} a_{2}=r$. If this diameter is an essential diameter we are done. If $a_{1} a_{2}$ is an extraneous diameter, there is an essential diameter parallel to it by Theorem 3; whence by Theorem 2 we deduce the existence of a pair of maximal parallel line segments $b_{1} b_{2}$ and $b_{3} b_{4}$ in the boundary of $C$ containing the points $a_{1}$ and $a_{2}$ respectively. As before (see Fig. 3) we take $b_{1} b_{2} b_{3} b_{4}$ in a counterclockwise sense on the boundary of $C$. Let the intersection of $b_{1} b_{3}$ and $b_{2} b_{4}$ be $b_{0}$. Now if $x$ is in either closed triangle $b_{0} b_{3} b_{4}$ or $b_{0} b_{1} b_{2}$ there is clearly an essential diameter which $x$ divides in the ratio $r$ with its respective end points on $b_{1} b_{2}$ and $b_{3} b_{4}$. If $x$ is outside these triangles it must still be in the closed trapezoid $b_{1} b_{2} b_{3} b_{4}$. Suppose it is in the triangle $b_{0} b_{4} b_{1}$ (Fig. 3 ). (It could be on $b_{1} b_{4}$.) Now, extend $b_{4} x$ through $x$ until it meets $b_{1} b_{2}$ in $a_{3}$ to the "left" of $b_{2}$. Consider any chord of $C$ through $x$ with one end point between $a_{3}$ and $b_{2}$. $x$ cannot divide this chord in a ratio less than $r$ or the other end point would fall "above" the extension of $b_{3} b_{4}$ through $b_{4}$ violating convexity, nor can it divide the chord in the ratio $r$ for then its end points would be on the extension of $b_{3} b_{4}$ and $b_{3} b_{4}$ is a maximal line segment. Hence $x$ must divide any such chord in a ratio greater than $r$ contrary to our supposition that $r$ is a maximum. Hence $x$ must be in one of the closed triangles $b_{0} b_{3} b_{4}$ or $b_{0} b_{1} b_{2}$ and it divides an essential diameter in the ratio $r$.

REMARKs. The essential diameters all pass through the center of a central convex body. It may be shown that the critical point of a convex body divides at least three essential diameters in the critical ratio.

5. Properties of associated sets. Some basic properties of the associated sets are stated in the following theorem.

THEOREM 5. The associated sets $C(r)$ of a closed planar convex body have the following properties.

1. $C(r)$ is a closed convex set.

2. The boundaries of $C(r)$ simply cover the entire plane.

\footnotetext{
${ }^{6}$ The same type of proof given for external points in Appendix A applies nicely.
} 
3. $C(r)$ contains no points if $r<r^{*}$.

4. $C\left(r^{*}\right)$ contains only the critical point $x^{*}$.

5. $C\left(r_{1}\right) \supset C\left(r_{2}\right)$ if $r_{1}>r_{2}$.

6. If $r^{*} \leqq r<1$, the set $C(r)$ consists of precisely those points $x$ of $C$ such that $r(x) \leqq r$ and the boundary of $C(r)$ is determined by $r(x)=r$.

7. For $r^{*}<r<1$, consider a boundary point $x$ of $C(r)$. Let $a_{1} a_{2}$ be a diameter which $x$ divides in the ratio $r$ so that $a_{1} x / a_{1} a_{2}=r$. Then if $m_{1}$ through $a_{1}$ and $m_{2}$ through $a_{2}$ are parallel contact lines of $C$, there is a contact line of $C(r)$ through $x$ parallel to $m_{1}$.

8. For $r>1$, the convex body $C(r)$ may be described as the set of all points lying on diameters of $C$ extended about their midpoints by the ratio $2 r-1$.

9. For $r>1, C(r)$ is the minimal convex body such that $C$ consists of points dividing its chords in a maximum ratio no greater than $r /(2 r-1)$.

10. If $r_{1}>r_{2} \geqq 1$, then $C\left(r_{1}\right)$ is the minimal convex body such that $C\left(r_{2}\right)$ is comprised of points dividing the chords in a maximum ratio $\left(r_{1}+r_{2}-1\right) /\left(2 r_{1}-1\right)$.

11. The essential diameters of $C(r), r>1$, are extensions of the essential diameters of $C$ by the ratio $2 r-1$ about their midpoints.

12. The sets $C(r)$ are invariant under affine transformations of the plane.

6. Proof of Theorem $5(r<1)$. If $r<1$ we have defined the associated sets as set products of closed convex sets. Hence $C(r)$ is closed and convex (property 1). If $x$ divides a chord in a ratio greater than $r$, it is outside some $C_{b}(r)$, and if $x$ divides every chord in a ratio less than or equal to $r$, it is in every $C_{b}(r)$ and hence it is in $C(r)$. By continuity of $r(x)$ it follows that $r(x)=r$ on the boundary of $C(r)$. No point $x$ interior to $C(r)$ exists such that $r(x)=r$, as this equality implies that $x$ is on the boundary of a $C_{b}(r)$ where $b$ is an end point of a diameter of $C$ passing through $x$. Hence $x$ must be a boundary point of $C(r)$ if $r(x)=r$. This proves property 6 and also shows that the boundaries of $C(r)$ simply cover the interior of $C$ (property 2). If $r_{1}<r_{2}$, then $C_{b}\left(r_{1}\right) \supset C_{b}\left(r_{2}\right)$ for every boundary point $b$ of $C$. Hence $C\left(r_{1}\right) \supset C\left(r_{2}\right)$ (property 5). If $a_{1} a_{2}$ is a diameter of $C$ divided by a point $x$ in a ratio $r(x)=r$ where $a_{1} x / a_{1} a_{2}=r$, then $C_{a_{1}}(r)$ has a contact line $m$ through $x$ parallel to any parallel pair of contact lines $m_{1}$ and $m_{2}$ passing respectively through $a_{1}$ and $a_{2}$. This contact line $m$ is also a contact line of $C(r)$ as $C(r) \subset C_{a_{1}}(r)$ and $x \in C(r)$ (property 7). The affine invariance is obvious (property 12).

7. Proof of Theorem $5(r>1)$. For $r>1$ the proof of properties 5 and 12 is again trivial. However, the proof of the other properties is 
somewhat more lengthy so we shall give these in a series of lemmas.

LEMMA 1. If $m_{1}$ and $m_{2}$ are parallel contact lines of $C$ passing respectively through the end points $b_{1} b_{2}$ of a diameter of $C$, then the line segments $a_{1} a_{2}$ obtained by extending $b_{1} b_{2}$ about its midpoint with a ratio $2 r-1$ is contained in $C(r)(r>1)$ and the lines, $n_{1}$ and $n_{2}$ parallel to $m_{1}$ and passing through $a_{1}$ and $a_{2}$ respectively determine a closed strip containing $C(r)$, that is, $a_{1} a_{2}$ is a diameter of $C(r)$.

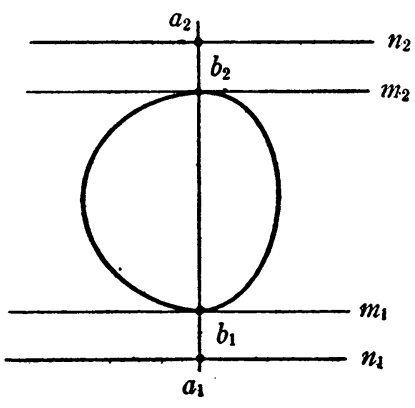

FIG. 4

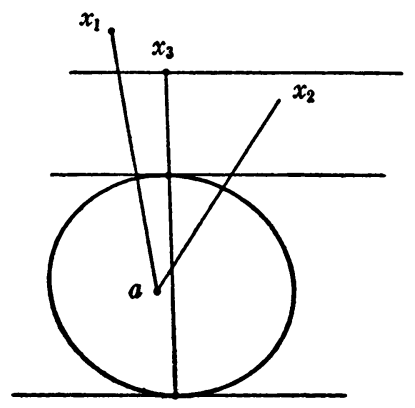

FIG. 5

Proof of Lemma 1. For a construction see Fig. 4. Since the line segment $b_{1} a_{2}$ is in $C_{b_{1}}(r)$ and $a_{1} b_{2}$ is in $C_{b_{2}}(r)$, the entire line segment $a_{1} a_{2}$ is in $C(r)$. However, since $C$ lies in the closed strip $m_{1} m_{2}$, no boundary point of $C(r)$ can be outside the closed strip $n_{1} n_{2}$. Hence $n_{1}$ and $n_{2}$ are parallel contact lines of $C(r)$.

LEMMA 2. $C(r)$ is closed and convex.

Proof of Lemma 2. Extend each essential diameter of $C$ about its midpoints with a ratio $2 r-1$. The end points of these extensions determine a simple closed curve which is the boundary of $C(r)$ and the set of all points on these diameters covers the closed region bounded by this curve (Theorem 3 ). Therefore $C(r)$ is closed. The convexity of $C(r)$ will follow if the boundary curve is convex. Let $x_{1}$ and $x_{2}$ be two points on this curve. If $x_{1} x_{2}$ is an extended essential diameter of $C$, by Lemma 1 all its points are in $C(r)$. Otherwise $x_{1}$ and $x_{2}$ are end points of two extended essential diameters of $C$ and these by Theorem 3 intersect in $C$ in a point $a$ (see Fig 5). Now if $x_{3}$ is a boundary point of $C(r)$, it cannot be interior to the triangle $x_{1} a x_{2}$; for if it were, there would be a contact line of $C(r)$ passing through it which would exclude either $x_{1}$ or $x_{2}$ from $C(r)$ (Lemma 1). Hence the boundary of $C(r)$ is convex.

Lemmas 1 and 2 complete the proof of properties 1 and 8 and also 
immediately imply the rest of the proof of property 2 . We next consider property 11.

Lemma 3. The essential diameters of $C(r), r>1$, are precisely the extensions of the essential diameters of $C$ with a ratio $2 r-1$ about their midpoints.

Proof of Lemma 3. We have already observed that all extensions of diameters of $C$ as indicated in Lemma 1 are diameters of $C(r)$. The assignment of essential diameters to $C(r)$ is unique unless $C(r)$ contains two parallel line segments in its boundary. It may be readily verified that, if $C(r)$ does contain two parallel line segments in its boundary, $C$ either has a line segment in its boundary or $C$ has two parallel line segments in its boundary, any one of these line segments being parallel to the ones on the boundary of $C$. Then, from our definition, essential diameters of $C(r)$ are extensions of the essential diameters of $C$.

Lemma 3 establishes property 11 and it forms the cornerstone for proofs of properties 9 and 10. It also lends further point to the term essential as applied to certain diameters.

Lemma 4. For $r>1, C(r)$ is the minimal convex body such that $C$ consists of points dividing chords of $C(r)$ in a maximum ratio no greater than $r /(2 r-1)$.

Proof of Lemma 4. Let $C(r)=B$ and consider the associated body $B(r /(2 r-1))$. Suppose $y$ is a boundary point of $B(r /(2 r-1))$. Then $y$ divides an essential diameter of $B$ in the ratio $r /(2 r-1)$ (Theorem 4$)$. However, the essential diameters of $B$ coincide with extensions of essential diameters of $C$ by Lemma 3 and thus $y$ is a boundary point of $C$. This implies that the boundaries of $B(r /(2 r-1))$ and $C$ coincide; hence $C=B(r /(2 r-1))$.

To prove that $B=C(r)$ is minimal with respect to the equality $B(r /(2 r-1))=C$, let $A$ be a convex body such that $A(r /(2 r-1))=C$. Consider the diameter $b_{1} b_{2}$ of $C$ and its extension $a_{1} a_{2}$ described in Lemma 1 (Fig. 3). The extension of $b_{1} b_{2}$ must intersect $A$ in two boundary points $c_{1}$ and $c_{2}$ such that $c_{1} c_{2}$ contains $b_{1} b_{2}$. Then the following inequality must be satisfied for $C=A(r /(2 r-1)), c_{1} b_{2} / c_{1} c_{2}$ $\leqq r /(2 r-1)$ and $b_{1} c_{2} / c_{1} c_{2} \leqq r /(2 r-1)$. This, however, implies that the line segment $c_{1} c_{2}$ contains $a_{1} a_{2}$. As $B=C(r)$ is the totality of points on extensions of diameters of $C$ about their midpoints with a ratio $2 r-1$ (Lemma 1), $B \subset A$, which concludes the proof of Lemma 4 which is property 9 .

It is now clear that $C\left(r_{1}\right)$ is obtained from $C\left(r_{2}\right)$ for $r_{1}>r_{2} \geqq 1$ by 
extensions of the essential diameters of $C\left(r_{2}\right)$ about their midpoints with a ratio $\left(2 r_{2}-1\right) /\left(2 r_{1}-1\right)$. Hence an application of Lemma 4 with $C$ replaced by $C\left(r_{2}\right)$ and $r=\left(r_{1}+r_{2}-1\right) /\left(2 r_{1}-1\right)$ gives property 10 and completes the proof of Theorem 5 .

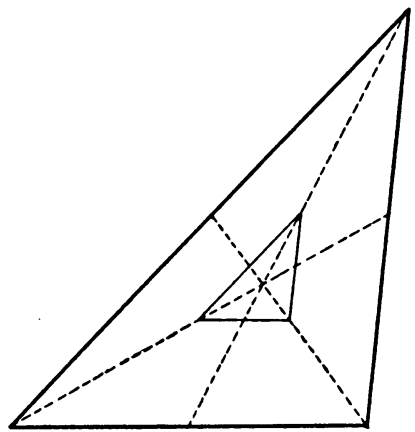

FIG. 6

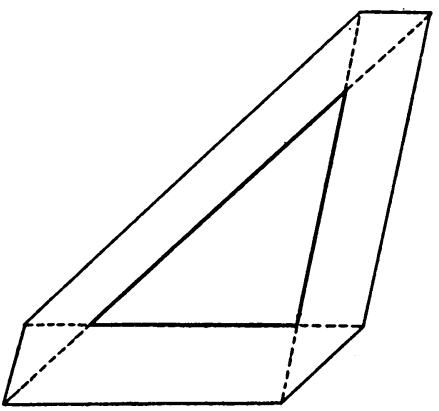

FIG. 7

8. Examples. To illustrate the principles stated in Theorems 1 and 2 and to provide examples for the next section, a few figures are shown here. Figure 6 shows a triangle and an associated body $C(r)$, $r<1$, which is a triangle. Observe that only three diameters of $C(r)$ lie on diameters of $C$. Figure 7 shows a triangle and an associated body $C(r)$ for $r>1$. The associated body $C(r)$ may be obtained by extending all diameters of the triangle about their midpoints with a ratio $2 r-1$. $C(r)$ is a hexagon with opposite sides parallel to the sides of the triangle.

Figure 8 is a semicircle $C$ with an associated body $C(r), r<1$. All diameters of the semicircle pass through either one of the two corner

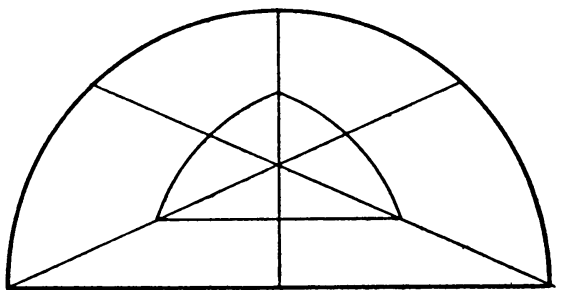

FIG. 8

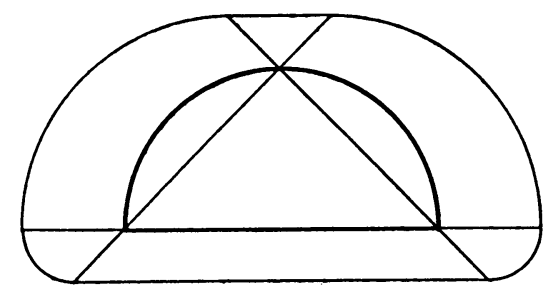

FIG. 9

points or the "vertex." One determines the critical point $x^{*}$ of the semicircle by determining the point on the chord of symmetry which divides diameters through $b_{1}, b_{2}$, and $b_{3}$ in the same ratio. This point is at a distance $2^{1 / 2}-1$ times the "radius" from the "center." Figure 
9 shows a semicircle $C$ and an associated set $C(r), r>1$. It is obtained by expanding the diameters of $C$.

9. Reducibility of convex bodies. Properties 9 and 10 of Theorem 5 show that one has a reversible type of transformation from $C$ to $C(r)$ for $r>1$, that is, if $B=C(r)$ then $C=B(r /(2 r-1))$. The example of the triangle in $\$ 8$ shows that the transformation from $C$ to $C(r)$ is not in general reversible if $r<1$, that is, if $B=C(r)$ then $C$ is not in general $B(r /(2 r-1))$ although $C \supset B(r /(2 r-1))$.

The following definitions will be justified where necessary in subsequent theorems. Let $B=C(r)$ for $r \leqq 1$. We say that a closed planar convex body $C$ is completely reducible if $C=B(r /(2 r-1))$ for every $r$ such that $r^{*}<r \leqq 1$. A convex body $C$ is said to be reducible to $C(r)$, $r^{*}<r \leqq 1$, if $C=B(r /(2 r-1))$ but $C \neq B\left(r^{\prime} /\left(2 r^{\prime}-1\right)\right)$ for any $r^{\prime}<r$. In particular, if $C$ is reducible to $C(1)=C$ we say $C$ is irreducible.

Theorem 6. A closed planar convex body $C$ is completely reducible if and only if the boundary of $C$ is a central curve $\left(r^{*}=1 / 2\right)$ and in that case all associated convex bodies $C(r)$ are obtained from $C$ by similitudes of $C$ about the center point $x^{*}$.

Proof of Theorem 6. Let $\epsilon>0$ be given. Then we may find an $r>r^{*}$ such that no point on the boundary of $C(r)$ is further from $x^{*}$ than $\epsilon$. The essential diameters of $C(r)$, by the definition of complete reducibility and property 11 of Theorem 5, extend by a fixed ratio about their centers to give the essential diameters of $C$. Hence the centers of all essential diameters of $C$ are arbitrarily close to $x^{*}$ and $x^{*}$ is the midpoint of all essential diameters. Hence $C$ has a central curve for a boundary. Now, properties 9, 10, and 11 of Theorem 5 immediately show that $C(r)$ is obtained from $C$ by extending or shrinking the essential diameters about their common midpoint $x^{*}$ with the ratio $2 r-1$.

THEOREM 7. If $r^{*} \neq 1 / 2$ for a closed planar convex body $C$, then there exists a unique ratio $r_{i}$ such that $C$ is reducible to $C\left(r_{i}\right)$ where $r^{*}<r_{i} \leqq 1$. $C(r)$ is irreducible.

Proof of Theorem 7. For any $r \leqq 1$ let $B=C(r)$. If $r_{1}<r_{2}$ and $C=B\left(r_{1} /\left(2 r_{1}-1\right)\right)$, then $C=B\left(r_{2} /\left(2 r_{2}-1\right)\right)$ by property 10 of Theorem 5. Furthermore, if $r_{1}<r_{2}$ and $C \neq B\left(r_{2} /\left(2 r_{2}-1\right)\right)$, then $C$ $\neq B\left(r_{1} /\left(2 r_{1}-1\right)\right)$. Since $r^{*} \neq 1 / 2$, from the above and Theorem 6 , it follows that there exists a unique ratio $r_{i}>r^{*}$ such that $C$ $=B\left(r_{2} /\left(2 r_{2}-1\right)\right)$ if $r_{2}>r_{i}$ and $C \neq B\left(r_{1} /\left(2 r_{1}-1\right)\right)$ if $r_{1}<r_{i}$. Now if $r_{i}=1$ we are finished. If $r_{i}<1$ we must show that $C=B\left(r_{i} /\left(2 r_{i}-1\right)\right)$. This follows from the fact that as $r$ approaches $r_{i}$ from above, the 
boundary of $C(r)$ is uniformly close to the boundary of $C\left(r_{i}\right)$ (Theorem $5)$ and the essential diameters of $C(r)$ lie on the essential diameters of $C$. Hence the essential diameters of $C\left(r_{i}\right)$ must lie along the essential diameters of $C$ and have the same midpoints. As $C=B(r /(2 r-1))$ for $r>r_{i}, C=B\left(r_{i} /\left(2 r_{i}-1\right)\right)$.

10. Concluding remarks. The definition of associated sets generalizes without alteration to $n$ dimensions. The uniqueness of the critical point $x^{*}$ we have not yet established for $n=3$. Results not involving $x^{*}$ for $r \leqq 1$ generalize to $n$ dimensions directly. The proofs of certain properties of associated sets $C(r)$ for $r>1$ hinged strongly on the notion of essential diameters which we defined for the plane case. We have defined essential diameters for three dimensions in a manner which will generalize, but we shall defer this generalization to a later paper. The convexity, closure, and covering properties of bodies $C(r)$ for $r>1$ can be readily established for $n$ dimensions without recourse to essential diameters. Hence we have a simple covering of $n$-dimensional space by a family of convex surfaces.

Many problems which may be new suggest themselves; we mention a few. Is the triangle characterized among convex planar bodies by the fact that its essential diameters sweep out a maximum of thrice the area of the body? Is the $n$-dimensional simplex characterized among $n$-dimensional convex bodies by the property that every interior point lies on precisely $n+1$ diameters $(n \geqq 2)$ ? The body $C(r)$ for $r>1$ has a minimal property with respect to $C$. What is its relationship to a maximal body if such exists?

Professor Andrew Sobczyk has recently shown that for $n \geqq 3$ critical points are not generally unique. ${ }^{7}$

The writer wishes to thank Charles A. Lehman for drawing the figures here presented.

Appendix A. The referee proposed the following neat proof of the statement referenced by footnote 4 .

Let $\max b_{1} b_{2} / b_{1} x=b_{1}^{*} b_{2}^{*} / b_{1}^{*} x=r$. Let $C_{x}(1-r)$ be the image of $C$ by the similarity with ratio $1-r$ about $x$. Then $C$ and $C_{x}(1-r)$ have $b_{2}^{*}$ but no interior points in common. Therefore $C$ and $C_{x}(1-r)$ have a separating contact line $m$ through $b_{2}^{*}$; moreover, there then exists a contact line of $C$ through $b_{1}^{*}$ parallel to $m$. Hence $b_{1}^{*} b_{2}^{*}$ is a diameter of $C$.

Appendix B. (Inserted on proof.) The author has extended

7 This result is contained in an unpublished manuscript by Professor Sobczyk and the writer entitled The critical points of a convex body. 
Theorem 5 to $n$ dimensions except that the statements concerning the critical points must be replaced by those concerning the critical set of points, $C\left(r^{*}\right)$, in view of Professor Sobczyk's examples, and property 11 is omitted in the present generalization. There also follow the same type of irreducibility considerations as given here. These extensions will be published soon.

Los Alamos Scientific Laboratory 